\title{
Efficient Method for Three Loop MMSE-SIC based Iterative MIMO Systems
}

\author{
Zuhaibuddin Bhutto ${ }^{1 *}$, Saleem Ahmed ${ }^{2}$, Syed Muhammad Shehram Shah ${ }^{3}$ \\ Azhar Iqbal $^{4}$, Faraz Mehmood ${ }^{5}$, Imdadullah Thaheem ${ }^{6}$, Ayaz Hussain ${ }^{7}$ \\ Computer System Engineering Department, Balochistan University of Engineering \& Technology, Pakistan ${ }^{1}$ \\ Computer System Engineering Department, Dawood University of Engineering \& Technology, Pakistan ${ }^{2}$ \\ Software Engineering Department, Mehran University of Engineering \& Technology, Pakistan ${ }^{3}$ \\ Deptartment of Basic Sciences and Mathematics, Dawood University of Engineering \& Technology, Pakistan ${ }^{4,5}$ \\ Energy Systems Engineering Department, Balochistan University of Engineering \& Technology, Pakistan ${ }^{6}$ \\ College of Information and Communication Engineering, SungkyunKwan University, Suwon, Republic of Korea ${ }^{7}$
}

\begin{abstract}
Iterative decoding is one of the promising methods to improve the performance of MIMO systems. In iterative processing channel decoder and MIMO detector share the information in order to enhance the overall system performance. However, iterative processing requires a lot of computations therefore it is considered as a computationally complex approach due to complex detection schemes involving iterative processing. There are several promising detection methods that require further improvements and they can be candidates in order to practically implement iterative processing. In this paper, the propose method to improve the efficiency of three loop-based minimum mean squared errors with soft interference cancellation (MMSE-SIC) method by reducing its complexity with a single inverse operation. Simulation results are given in order to provide detail analysis of the proposed MMSE-SIC based approach for iterative detection and decoding (IDD).
\end{abstract}

Keywords-MIMO; Iterative Detection and Decoding (IDD); sphere decoding; Minimum Mean Squared Errors with Soft Interference Cancellation (MMSE-SIC)

\section{INTRODUCTION}

To acquire a higher data rate, MIMO techniques are widely used in most current wireless communication systems. The channel coding or forward error correction (FEC) scheme is an important part of MIMO communication systems if one targets high QoS for mobile users. It is essential to exploit high-performance FEC methods to achieve the performance gains in MIMO based communication systems. The FEC methods like turbo codes and LDPC codes [1] promises to come close to the Shannon capacity limit. The harsh channel conditions demand to use FEC schemes with iterative decoding to achieve the performance goals. Turbo codes are one of the coding schemes that are based on the concept of iterative decoding [1].

The iterative decoding approach can be employed as the outer loop, which is a connection between the MIMO detector and FEC decoder. In such scenario, the iterative loop between the MIMO detector and FEC decoder utilizes extrinsic LLRs iteratively [2]-[4]; therefore, it is known as joint iterative detection and decoding (JIDD). One major implementation difficulty of the JIDD based MIMO systems is the signal detection issue at the receiving side and its computational complexity which makes it impractical.

Previous research has employed reduced search MAP methods and equalization method for SISO detectors. The sphere decoding and tree search-based methods have been very promising approaches for JIDD systems [4]-[6]. These approaches target to reduce the MAP search space by finding the likely candidates. However, tree search methods are still computationally complex. In [7][8], a minimum mean squared error with soft interference cancellation (MMSE-SIC) detector is derived from the MMSE detector with the interference cancelation as the pre-process by considering a priori information from the channel decoder. The MMSE-SIC detection method does not provide promising performance improvement in the JIDD system, but they benefit from low complexity. There have been considerable performance enhancements in the MMSE-SIC method. In [9], the author used two approaches to enhance the performance of the MMSE-SIC method. First, a posteriori information is used to enhance the performance of the MMSE-SIC method and complexity is reduced by hard decision threshold (HDT) method. However, still, there is a performance gap between MMSE-SIC and other existing methods like tree search-based methods. In [10] author employed a three-loop approach to further enhance the performance of the MMSE-SIC method. The third loop enhances the performance of the method with the expenses of additional processing. The main complexity of the MMSE-SIC method lies in its filtering matrix inversion method which is addressed in [11] by proposing a single matrix inversion method.

In this paper, the proposed method is used to reduce the complexity of the three-loop method which involves repeated filtering matrix inversion for each transmitted layer. The purpose to use a single inversion of filtering matrix for all layers in the three-loop method. Simulation results show that the proposed method is more suitable with negligible performance degradation with only single inversion instead of layer-based inversion of the filtering matrix.

The rest of the research paper is outlined as follows. The three-loop method is explained with its system diagram in Section II. Then Section III is about the proposed single

*Corresponding Author 
inversion approach for the three-loop MMSE-SIC method. Section IV is about simulation results. Finally, the paper is concluded in the conclusion section.

\section{CONVENTIONAL DETECTION METHODS FOR JIDD SYSTEMS}

The system diagram of the conventional JIDD based MIMO system is shown in Fig. 1. The MIMO system of size is considered. The system diagram shows that information bits, $\mathbf{u}$ are sent to FEC encoder to produce the coded output represented as $\mathbf{u}$. The size of each codeword length is of $n$. Then bit interleaving is done with codewords having a size of $M \square K$ resulting in interleaved bits $\mathbf{x}$.

Then the bits are divided into MIMO frames of size $M \square K$ bits. Each MIMO frame containing transmitting bits can be represented as:

$\mathbf{S}=\left[s_{1,1}, \cdots, s_{1, K}, s_{2,1}, \cdots, s_{M, K}\right]$

where $S_{m, k}$ is the kth bit which is mapped onto the $m^{\text {th }}$ transmitting symbol.

The transmitted information vector is represented as, $\mathbf{x}=\left[x_{1}, x_{2}, \cdots, x_{M}\right]^{T}$, where symbols are from constellation, $\Omega, \mathbf{x} \in \Omega^{M}$. The received information vector is represented as, $\mathbf{y}=\left[y_{1}, y_{2}, \cdots, y_{M}\right]^{T}$, it can be represented with an $N \times M$ complex channel matrix, as follows:

$\mathbf{y}=\mathbf{H x}+\mathbf{n}$

where $n$ is an $N \times 1$ complex Gaussian noise vector. The entries of channel matrix $\mathbf{H}$ are assumed to be known at the receiver.

There are several existing detection methods which are employed in JIDD system. The optimum soft MAP demodulator calculates the exact LLR values in JIDD systems. However it is impractical to use it due to its computational complexity. The LSD is also one of the methods to generate soft information based on SD algorithm. Another approach which searches for the ML solution and its counter hypothesis for soft output is STS [5]. Below are the details of existing detection methods employed in JIDD systems.

\section{A. MAP based Detection for JIDD System}

Fig. 1 illustrates the overall MIMO detector system divided into transmitter and receiver. As depicted in Fig. 1, at receiver, first the soft bit information (SBI), $L$, is estimated from $y$ for all transmitted bits. Using the MAP detection process, $L\left(x_{m, k}\right)$, is calculated as:

$$
L\left(x_{m, k}\right)=\ln \left(\frac{P\left(x_{m, k}=+1 \mid \mathbf{y}\right)}{P\left(x_{m, k}=-1 \mid \mathbf{y}\right)}\right)
$$

where, $k, m$ are the $k^{\text {th }}$ bit of the $m^{\text {th }}$ symbol for which SBI information is calculated.

By applying Bayes' theorem, and use of interleaving operation which makes all information bits in a symbol vector statistically independent and under the max-log approximation, the $L\left(x_{m, k}\right)$ can be represented as [4]:

$L\left(x_{m, k}\right)=\ln \left(\frac{\sum_{\mathbf{s} \in \chi_{m, k}^{+1}} P(\mathbf{y} \mid x) \cdot P(x)}{\sum_{\mathbf{s} \in \chi_{m, k}^{-1}} P(\mathbf{y} \mid x) \cdot P(x)}\right) \approx \max _{x \in \chi_{m, k}^{+1}} d_{x}-\max _{x \in \chi_{m, k}^{-1}} d_{x}$

where $d x$ can be found as follows:

$d_{x}=-\frac{1}{N_{0}}\|\mathbf{y}-\mathbf{H} \mathbf{x}\|^{2}+\frac{1}{2} \sum_{m, k} x_{m, k} L_{a}^{d}\left(x_{m, k}\right)$.

The performance of the JIDD system employing MAP detection method is illustrated in Fig. 2. The performance is generated for $2 \times 2$ and $4 \times 4$ MIMO detector with 16 QAM modulation scheme. The performance is compared for different outer iterations which are between decoder and MIMO detector. The code rate of $1 / 3$ is set for the encoding and decoding purposes with the constraint length of 3 is set for each RSC component. The turbo decoder was employed with 8 inner iterations. As it can been seen that as outer iterations increases the performance of $4 \times 4$ system approaches $2 \times 2$ MIMO system.

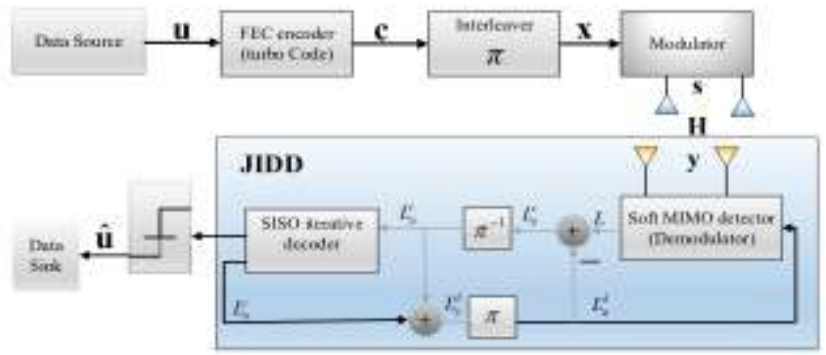

Fig. 1. Transmitter and Receiver Blocks for MIMO System with JIDD.

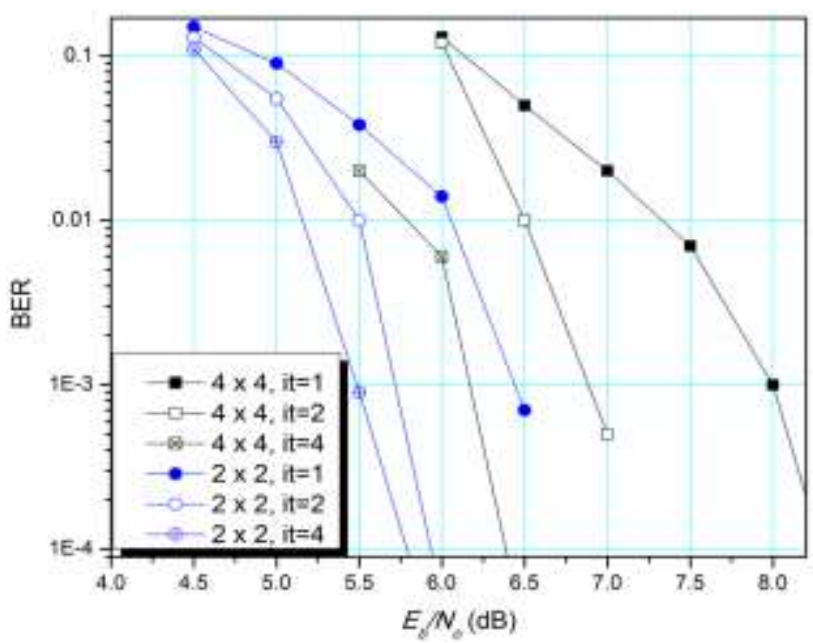

Fig. 2. BER Performance of JIDD based MIMO System Employing MAP Detector for the $2 \times 2$ and $4 \times 4$ System. 


\section{B. Sphere Decoding Based Detection Methods}

The SD scheme aims to search the transmitted information by searching the ML metric with minimum distance from received signal, which is considered as the final solution metric [4][12-14]. SD is a suboptimal solution to find the best solution by considering small space vector within a set radius instead of searching whole space of possibilities in a full space. The LSD is the method which is based on SD algorithm. In LSD, the search is not limited for one best solution which is the case in SD method, the LSD builds a list of Nc best solutions by searching through the space of radius $r$. The candidates whose radius falls inside $r$ whose are used to build a subset list $L_{L S D} \subset \chi$ of size Nc [6]. Even the list is full, LSD method continues its search for better candidates until it reaches the end of the search space. The LSD method performance varies for different sizes of $\mathrm{Nc}$, therefore, the increased size of candidate the performance of LSD methods enhances. However, a large size of $\mathrm{Nc}$ will result in higher candidate search complexity due to increase in search space.

Fig. 3 shows the BER performance [14-16] of Conventional JIDD detection methods over $2 \times 2$ MIMO system with a 16-QAM scheme. The turbo decoder is set with 8 inner iterations and there are 3 outer iterations performed between decoder and MIMO detector. The full search MAP can produce optimal performance compared to LSD and STS methods. Compared to the conventional LSD with 40 candidates, the STS method produces better performance. However performance of LSD depends on the list size which can be improved with increase in list size with the cost of computational complexity.

\section{Three Loop MMSE-SIC Method}

As depicted in Fig. 4, loops 1 and 2 are those which the JIDD system conventionally employs. In order to reduce the error propagation (EP) which occurs in MMSE-SIC based JIDD systems due to utilization of soft information of other layers, the additional Loop 3 is used and it can enhance the error-rate performance by reflecting soft information within the MIMO detector; so it uses 1) a priori soft information feedback by the FEC decoder, $L_{a}^{d}$; 2) a posteriori soft information which is generated from the first $L^{0}$ and second $L^{1}$ inner MMSE-SIC iterations. During the execution of Loop 3 , the MIMO detector estimates $L^{0}$ and it is stored so that it can be utilized in the second inner MMSE-SIC iteration jointly with $L^{1}$. While SIC-MMSE detector performing the first inner iteration, the $i^{\text {th }}$ streams soft symbol is calculated by utilizing $L_{a}^{d}$ and $L^{0}$ [10]. After completing the first iteration, generated soft information is feedback within the MMSE-SIC detector, both soft information extracted in the first MMSE-SIC iteration, and the a posteriori soft information $L^{1}$ found at the second MMSE-SIC process, are incorporated. This process continues with the detector until there is no further change in performance and finally generated a posteriori information in the last iteration is deinterleaved and forwarded to the channel decoder [10].

In Fig. 5, the impact of Loop 3 which is inside MMSE-SIC detector is elaborated. The third loop utilizes more reliable information during different steps involved in MMSE-SIC method. Fig. 2 illustrates the impact of Loop 3 on BER performance of $4 \times 4$ MIMO system. The number shown in parentheses represents the total layers utilizing information of previous detection iteration. In Fig. 5, the "3 loop method (1)" where (1) indicates information from previous iteration of Loop 3 is utilized only at first layer during current detection process. Similarly, (2) indicates that information is utilized in 2 current layers and so on. Therefore, as more layers use the information of previous loop from Loop 3, the BER performance is improved because information of previous layers is more reliable and it can reduce the error propagation resulting in performance enhancement.

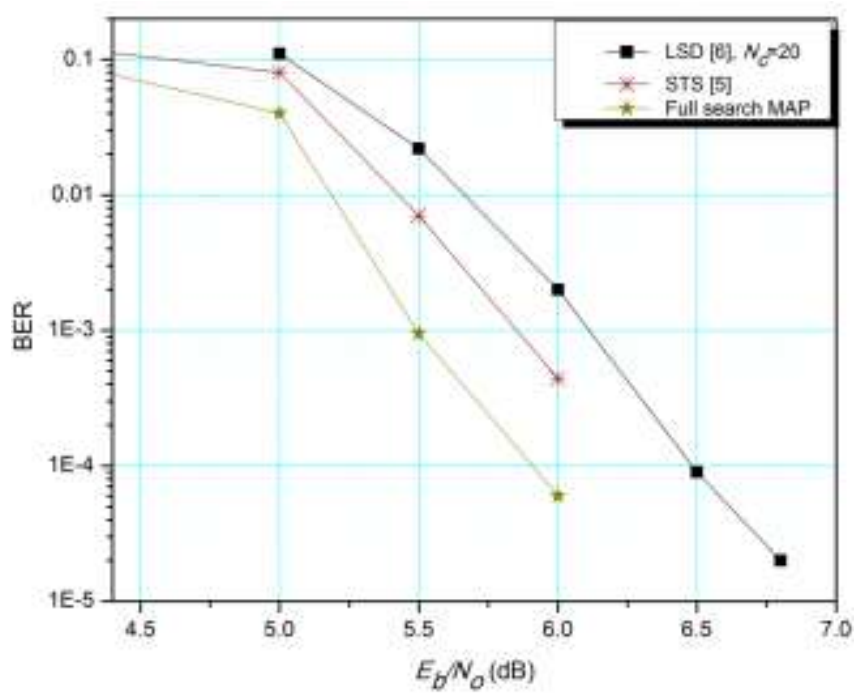

Fig. 3. BER Performance of Various Nonlinear JIDD Detection Methods Over $2 \times 2$ MIMO System with 16-QAM Modulation.

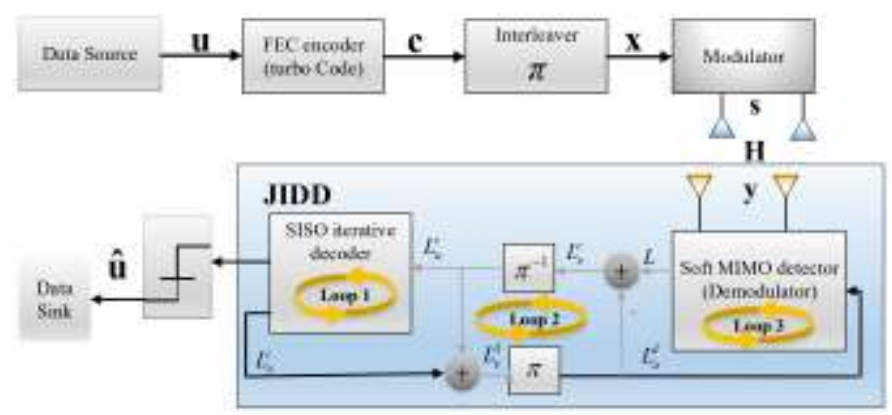

Fig. 4. MMSE-SIC based JIDD System having Three Iterative Loops. 


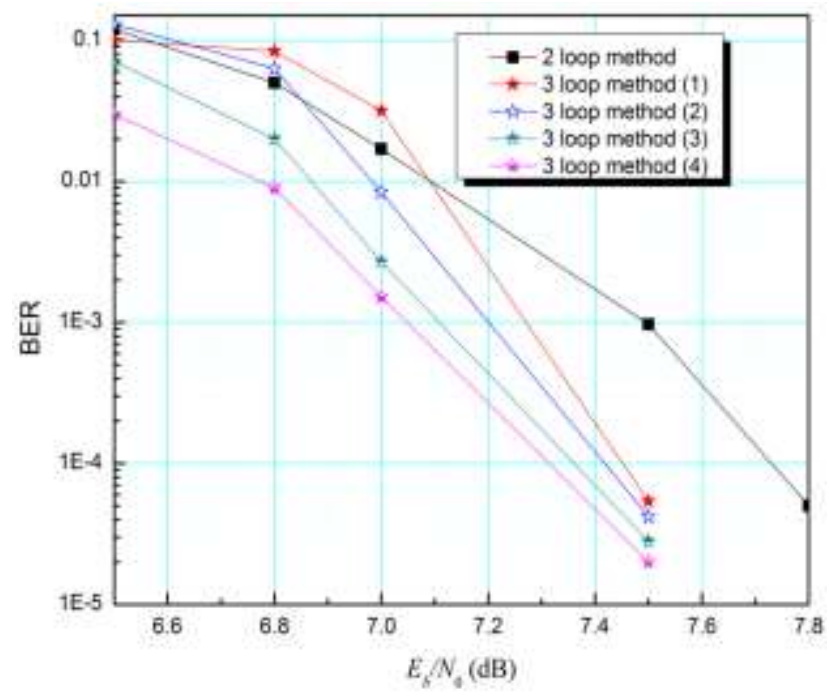

Fig. 5. BER Performance of Three Loop Method for $4 \times 4$ MIMO System.

\section{Single INVERSION BASED THREE LOOP METHOD}

Three loop approach for MMSE-SIC scheme can improve the performance but the cost of computational complexity. Each iteration in the third loop involves $M$ inverse operations of the filtering matrix. The single inversion method [11] can be employed together with three loops MMSE-SIC method to improve the overall efficiency of the system in terms of computational complexity with a negligible impact on performance. In this paper, the proposed method is used to reduce the complexity of the three-loop MMSE-SIC method by performing the filtering operation with a single inversion for each loop three iterations. By using the single filtering matrix inversion in each iteration of loop 3, the large amount of complexity is reduced while keeping the advantage of performance enhancement brought by the loop 3 approach. During the lth iteration inside Loop 3, the soft symbols $\tilde{x}_{j}^{l}$ for each later are estimated by utilizing different soft information in each of its iterations.

For the first iteration when $1=0$, the soft symbols are calculated using, which is soft information (a priori) provided by the channel decoder. During the first iteration in loop 3, the soft symbols for $j=1 ; \cdots M$; are found as:

$\tilde{x}_{j}^{0}=E\left[x_{i}\right]=\sum_{q \in \Omega} q \prod_{k=1}^{K} \frac{1}{2}\left(1+\tilde{s}_{j, k} \tanh \left(L_{a}^{d}\left(s_{j, k}\right)\right)\right)$

where $\tilde{s}_{j, k}$ is put as \pm 1 , which depend on the complex symbol $q$ taken from the complex space $\Omega$. The error, $e_{j}=x_{j}-\tilde{x}_{j}$, which can impact the overall performance of the system due to its impact in error propagation is the error calculated between the transmitted stream $x_{j}$ and its soft symbol $\tilde{x}_{j}$. The variance of each soft symbol depicts its reliability, which is found using [7][8]:

$\mathrm{V}[j, 0]=E\left[\left|e_{j}^{0}\right|^{2}\right]=\sum_{q \in \Omega}|q|^{2} \prod_{k=1}^{K} \frac{1}{2}\left(1+\tilde{s}_{j, k} \tanh \left(L_{a}^{d}\left(s_{j, k}\right)\right)\right)-\left|\tilde{x}_{j}^{0}\right|^{2}$.
In loop 3, only a priori information is used for soft symbol and their variance calculation. In the successive iterations, $1>0$, soft symbols and their variance are found by using both a posteriori information of previous loop 3 iterations. Therefore, for successive iteration, found as:

$\tilde{x}_{j}^{l}=\sum_{q \in \Omega} q \prod_{k=1}^{K} \frac{1}{2}\left(1+\tilde{s}_{j, k} \tanh \left(L^{l-1}\left(s_{j, k}\right)-L_{a}^{d}\left(s_{j, k}\right)\right)\right)$

and their variance is calculated using:

$\mathrm{V}[j, l]=E\left[\left|e_{j}^{l}\right|^{2}\right]=\sum_{q \in \Omega}|q|^{2} \prod_{k=1}^{K} \frac{1}{2}\left(1+\tilde{s}_{j, k} \tanh \left(L^{l-1}\left(s_{j, k}\right)-L_{a}^{d}\left(s_{j, k}\right)\right)\right)-\left|\tilde{x}_{j}^{l}\right|^{2}$.

After calculating the soft symbols and their variance we estimate Gram matrix and matched filter output. The interference cancelation for the layer is done using:

$\tilde{\mathbf{y}}_{i}^{l}=\mathbf{y}^{M F}-\sum_{j, j \neq i} \mathbf{g}_{j} \tilde{x}_{j}^{l}=\mathbf{h}_{i} x_{i}+\tilde{\mathbf{n}}_{i}^{l}$,

where $\tilde{\mathbf{n}}_{i}^{l}=\sum_{j, j \neq i} \mathbf{h}_{j} \boldsymbol{e}_{j}^{l}+\mathbf{n}$, and $\mathbf{g}_{j}$ is the $j^{\text {th }}$ column of $G$, and $\tilde{x}_{i}^{l}=\left[\tilde{x}_{1}^{l}, \cdots, \tilde{x}_{i-1}^{l}, 0, \tilde{x}_{i+1}^{l}, \cdots, \tilde{x}_{M}^{l}\right]^{T}$.

The next step is filtering the MMSE matrix which involves $M$ inverse operation (one for each layer). In order to perform single inversion for each iteration in the loop 3 , we use the layer independent variance matrix,

$\sum^{l}=\operatorname{diag}\{V[1, l], \cdots, V[M, l]\}$.

Then the MMSE filtering matrix, $\mathbf{W}^{l}$, is calculated only once in each iteration of loop 3 by using:

$\mathbf{W}^{l}=\left(\mathbf{G} \sum^{l}+\sigma^{2} \mathbf{I}_{N}\right)^{-1}$.

It is noticeable that previously three-loop approach for MMSE-SIC used (13) during the filtering process which is the layer dependent variance matrix and is found as:

$\sum_{i}^{l}=\operatorname{diag}\{V[1, l], \cdots, V[i-1, l], 1, V[i+1, l], \cdots, V[M, l]\}$.

The symbol, $\hat{x}_{i}^{l}$ is calculated by using:

$\hat{x}_{i}^{l}=\left(\mathbf{w}_{i}^{l}\right) \tilde{\mathbf{y}}_{i}^{l}$.

The symbol estimation in equation (14) can be expended as:

$\hat{x}_{i}^{l}=\mu_{i}^{l} x_{i}+\eta_{i}^{l}$

where

$\mu_{i}^{l}=\left(\mathbf{w}_{i}^{l}\right) \mathbf{g}_{i}$

and denotes Gaussian random variable having a variance $\left(\tilde{\sigma}_{i}^{l}\right)^{2}$, which is obtained by using:

$\left(\tilde{\sigma}_{\mathbf{i}}^{1}\right)^{2}=\mu_{i}^{l}-\left|\mu_{i}^{l}\right|^{2}$ 
The further complexity can be reduced by using a single distance calculation method in order to find the soft information for each bit. The hard decision threshold (HDT) scheme is an efficient single distance calculation method which can calculate of the kth bit using [9]:

$$
L\left(s_{i, k}\right) \approx-2 \rho_{i}^{l} \tilde{b}_{k}^{l}
$$

where $\tilde{b}_{k}^{l}$ depicts the distance between the estimated symbol, and the HDT line for the estimating bit, and is the signal-to-interference-plus-noise ratio (SINR) for the layer.

\section{Simulation RESUlts}

Performance of the proposed method is compared for different MIMO systems over the 16-QAM modulation scheme. The Rayleigh fading channel model is deployed to evaluate the performance of the proposed method. The code rate of the turbo code is $1 / 3$. The constraint length employed was three for each recursive systematic convolutional (RSC) code. Eight iterations are deployed in the turbo decoder. The exchange of information between turbo decoder and MIMO detector was performed 4 times.

Fig. 6 illustrates the performance comparison of a single inversion based three-loop MMSE-SIC method with other conventional methods for a $2 \times 2$ MIMO system with a 16QAM modulation technique. The proposed method has outperformed conventional MMSE-SIC method while it has similar performance as conventional loop 3 method while much less complex because of single inversion in each iteration while conventional loop 3 requires $\mathbf{M}$ inversion operations in each iteration.

Fig. 7 illustrates the performance comparison of a single inversion based three-loop MMSE-SIC method with other conventional methods for a $4 \times 4$ MIMO system with a 16QAM modulation technique. As shown in Fig. 7, the proposed method has outperformed the conventional MMSE-SIC method while it has similar performance as the conventional loop 3 method while much less complex because of single inversion in each iteration while conventional loop 3 requires $M$ inversion operations in each iteration.

\section{A. Discussion on Complexity}

The complexity of the conventional MMSE-SIC method is dominated by its filtering operation which requires $M$ inverse operations in each iteration. The number of matrix inverse operations is directly proportional to the number of antennas. As the antenna size increases so do the number of matrix inversion operations. Therefore, the proposed method has no impact on the increase in the antenna size because it is layer independent which means it requires only a single inversion operation. Referring to Fig. 7, the conventional loop 3 method in [10] requires eight matrix inversion operation for 2 iterations while the proposed scheme needs only two matrix inversion operation (one for each iteration). Additionally, instead of Max-Log MAP approximation for SBI estimation, we employ a single distance calculation approach which further reduces the complexity of the proposed method.

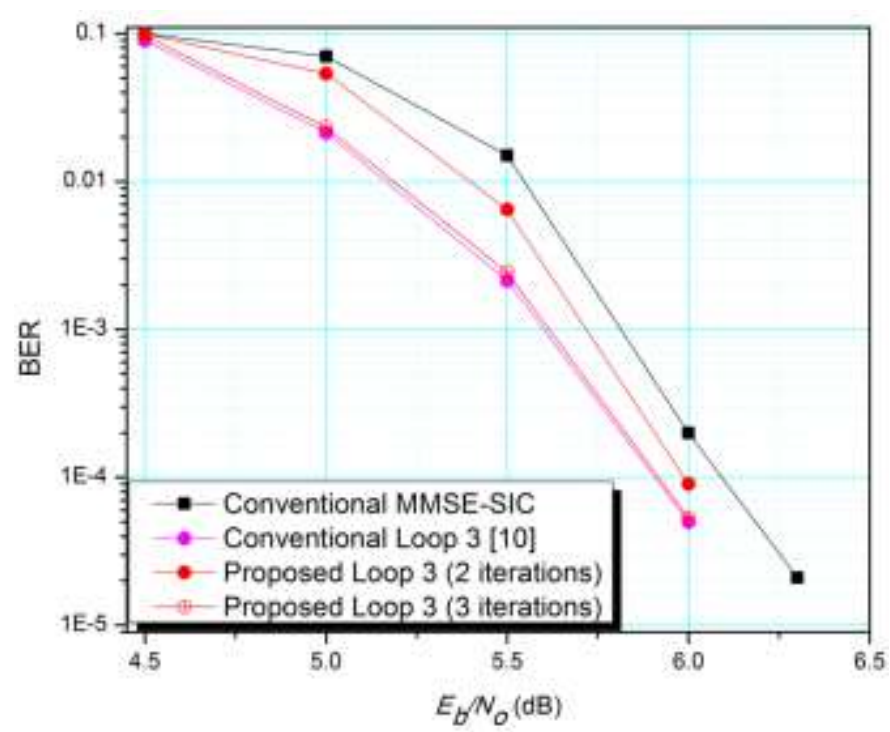

Fig. 6. BER Performance of Proposed Single Inversion based Three-Loop MMSE-SIC Method for a MIMO System with 16-QAM Modulation.

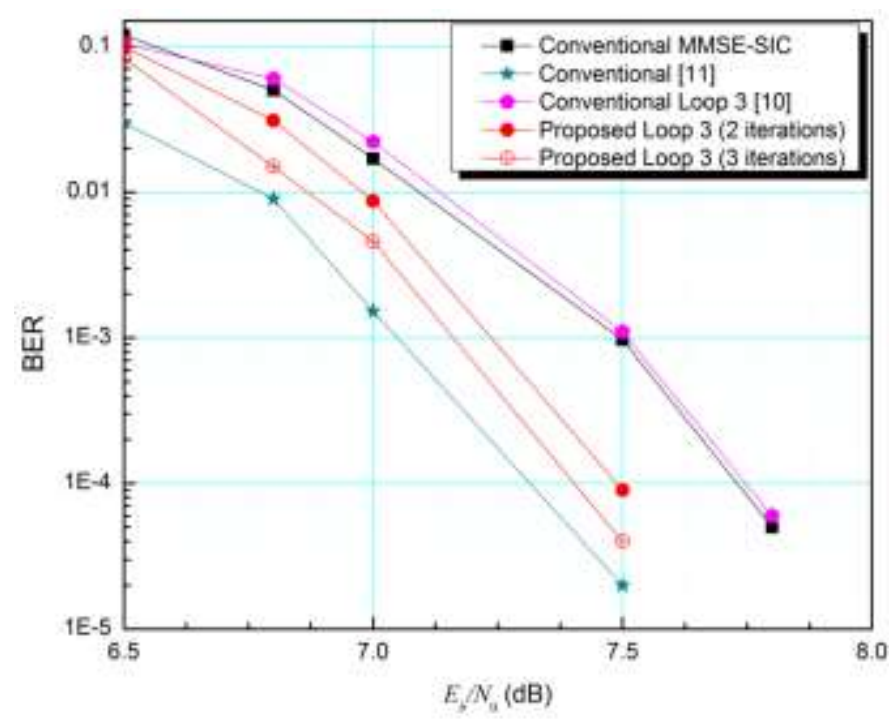

Fig. 7. BER Performance of Proposed Single Inversion based Three-Loop MMSE-SIC Method for a $4 \times 4$ MIMO System with 16-QAM Modulation.

\section{FINDINGS AND CONCLUSION}

Due to the large computational complexity of multiple matrix inversion in three loop MMSE-SIC method, the proposed single inversion method for three loop-based MMSE-SIC method. The proposed method improves the efficiency of the MMSE-SIC method by lowering the computational complexity. The simulation results are given which shows that the proposed method can improve the performance in each Loop 3 iteration with much lower complexity.

\section{ACKNOWLEDGMENT}

This work was supported by the Balochistan University of Engineering and Technology, Khuzdar Pakistan, Research Fund. 


\section{REFERENCES}

[1] C. Berrou, A. Glavieux, and P. Thitimajshima, "Near Shannon limit error-correcting coding and decoding turbo codes", in Proc. of the IEEE Intern. Conf. on Communications (ICC), Geneva, USA, pp. 1064-1070, May 1993.

[2] N. Khan, S. Ahmed, D. M. Saqib, "Study of MIMO Detection schemes for Emerging Wireless Communications", in International Journal of Computer Science and Network Security, vol.18 no.3, Mar. 2018.

[3] M. Zhang, S. Ahmed and S. Kim, "Iterative MMSE based Soft MIMO Detection with Parallel Interference Cancellation" in IET Communications, vol. 11, no. 11, pp. 1775-1781, Sept. 2017.

[4] B. M. Hockwald, and S. Ten Brink, "Achieving near-capacity on a multiple-antenna channel", IEEE Transaction Communication, vol. 51, no. 3, pp. 389-399, Apr. 2003.

[5] C. Studer, H. Blcskei, "Soft-input soft-output single tree-search sphere decoding”, IEEE Transactions on Information Theory, vol. 56, no. 10 pp. 4827-4842, Oct. 2010.

[6] S. Ahmed, S. Kim, "Efficient list-sphere detection scheme for joint iterative multiple-input multiple-output detection", IET Communication, vol. 8 , no. 18 , pp. 3341-3348, Dec. 2014.

[7] X. Wang, and H. V. Poor, "Iterative (turbo) soft-interference cancellation and decoding for coded CDMA", IEEE Transaction on Communication, vol. 47, no. 7, pp. 1046-1061, Jul. 1999.

[8] M. T. Tuchler, A. C. Singer, and R. Koetter, "Minimum mean squared error equalization using a priori information", IEEE Transaction on Signal Processing, vol. 50, no. 3, pp. 673-683, Mar. 2002.
[9] S. Ahmed, S. Kim, "Efficient soft bit estimation for joint iterative multiple-input multiple-output detection", IET Communications, vol. 9, no. 17, pp. 2107-2113, Nov. 2015.

[10] S. Ahmed and S. Kim, "Efficient SIC-MMSE MIMO detection with three iterative loops", International Journal of Electronics and Communications, vol. 72, pp. 65-71, Feb. 2017.

[11] C. Studer, S. Fateh, D. Seethaler, "ASIC implementation of soft input soft-output MIMO detection using MMSE parallel interference cancellation", IEEE Journal of Solid-State Circuits, vol. 46, no. 7, pp. 1754-1765, Jul. 2011.

[12] H. Vikalo and B. Hassibi, "On the expected complexity of sphere decoding", in the Thirty-Fifth Asilomar Conf. on Signals, Systems and Computers, vol. 2, pp. 1051-1055, Nov. 2001, Pacific Grove, CA, USA.

[13] O. M. Damen, H. E. Gamal, and G. Caire, "On maximum-likelihood detection and the search for the closest lattice point", IEEE Transactions on Information Theory, vol. 49, no. 10, pp. 2389-2402, Oct. 2003.

[14] Z. Bhutto, et at., "Efficient Method for SBI Estimation in Iterative Coded MIMO Systems", International Journal of Computer Science and Network Security (IJCSNS), vol. 19, no. 6, pp. 135-140, June 2019.

[15] A. Hussain, Z. Bhutto, "BER Performance of Opportunistic Relaying with Direct Link using Antenna Selection”, International Conference on Information Technology (AIT), Bangkok, Thailand, June 2012.

[16] A. Hussain, Z. Ahmed, and Z. Bhutto, "BER Performance of Opportunistic Relaying Scheme using Antenna Selection", 6th Joint IFIP Wireless and Mobile Networking Conference (WMNC 2013), Dubai, UAE, April 2013. 